\title{
Discovery of potent charge-reducing molecules for native ion mobility mass spectrometry studies
}

Jixing Lyu ${ }^{1} \neq$, Yang Liu ${ }^{1} \neq$, Jacob W. McCabe ${ }^{1, \ddagger}$, Samantha Schrecke ${ }^{1}$, Lei Fang ${ }^{1}$, David H. Russell ${ }^{1}$, Arthur Laganowsky ${ }^{1, *}$

${ }^{1}$ Department of Chemistry, Texas A\&M University, College Station, TX 77843

¥These authors contributed equally to this work

*Corresponding Author: alaganowsky@chem.tamu.edu

\section{CONTENTS}

- Table S1 $\mathrm{EC}_{50}$ values

- Table S2 Names and abbreviations

- Table S3 Total number of charge states

- Figure S1 AmtB in C8E4 without charge reducing agents

- Figure S2 AmtB in DDM with some charge reducing agents

- Figure S3 Example soluble protein MS data with charge reducing agents

- Figure S4 Arrival time distributions of AmtB by FT-IM-PF-DT

- Figure S5 Collision cross section plots of AmtB

- Figure S6 Lysozyme with spermine at different collision energy

- Figure S7 Lysozyme with spermine at different cone voltage

- Figure $\mathbf{S 8}$ Charge reduction of $\mathrm{AmtB}$ at different solution temperature 


\section{Supplementary Tables}

Table S1. $\mathrm{EC}_{50}$ and other fitting parameters for different charge-reducing molecules.

\begin{tabular}{l|l|l|l|l|} 
& $\mathbf{n}_{\mathbf{H}}$ & $\mathbf{E C}_{\mathbf{5 0}}(\mathbf{m M})$ & $\boldsymbol{b}$ & $\mathbf{Z}_{\text {avg,min }}$ \\
\hline Trimethylamine N-oxide & 1.1 & 87.3 & 6.5 & 7.9 \\
\hline $\begin{array}{l}\text { Histamine } \\
\text { Spermidine }\end{array}$ & 1.1 & 8 & 2.1 & 12 \\
\hline $\begin{array}{l}\text { Spermine } \\
\text { Piperazine }\end{array}$ & 1.7 & 12.9 & 3.1 & 10.8 \\
\hline $\begin{array}{l}\text { 1-(2-Aminoethyl)piperazine } \\
\text { 1,4-Bis(3-aminopropyl)piperazine }\end{array}$ & 1.5 & 10.2 & 3.9 & 9.9 \\
\hline $\begin{array}{l}\text { Bis(2-aminoethyl)amine } \\
\text { Bis(3-aminopropyl)amine }\end{array}$ & 1.3 & 8.2 & 1 & 13.9 \\
\hline $\begin{array}{l}\text { 3,3-Diamino-N-methyldipropylamine } \\
\text { Tris(2-aminoethyl)amine }\end{array}$ & 1.1 & 6.8 & 1.5 & 13 \\
\hline Tris(3-aminopropyl)amine & 1.5 & 11.6 & 3.4 & 10.4 \\
\hline & 1.2 & 14.2 & 1.7 & 12.6 \\
\hline & 0.8 & 10.4 & 2.4 & 11.6 \\
\hline & 0.9 & 18.6 & 2.9 & 11 \\
\hline
\end{tabular}


Table S2. Name abbreviation of charge reducing chemicals.

\begin{tabular}{|c|c|}
\hline Chemicals & Abbreviation \\
\hline Trimethylamine N-oxide & TMAO \\
\hline 1-(2-Aminoethyl)piperazine & AEP \\
\hline 1,4-Bis(3-aminopropyl)piperazine & APP \\
\hline Bis(2-aminoethyl)amine & B2A \\
\hline Bis(3-aminopropyl)amine & B3A \\
\hline 3,3-Diamino-N-methyldipropylamine & DMP \\
\hline Tris(2-aminoethyl)amine & T2A \\
\hline Tris(3-aminopropyl)amine & T3A \\
\hline
\end{tabular}


Table S3. Total number of charge states $\left(Z_{\text {tot }}\right)$ of AmtB mixed with charge reducing agents at various concentrations.

\begin{tabular}{|c|c|c|c|c|c|c|}
\hline & $0 \mathrm{mM}$ & $10 \mathrm{mM}$ & $25 \mathrm{mM}$ & $50 \mathrm{mM}$ & $100 \mathrm{mM}$ & $250 \mathrm{mM}$ \\
\hline TMAO & 10 & 13 & 12 & 9 & 9 & 6 \\
\hline Histamine & 10 & 9 & 8 & 5 & 5 & 3 \\
\hline Spermidine & 10 & 10 & 5 & 5 & 5 & 4 \\
\hline Spermine & 10 & 11 & 8 & 5 & 3 & 3 \\
\hline Piperazine & 10 & 9 & 8 & 7 & 7 & 7 \\
\hline AEP & 10 & 7 & 7 & 7 & 7 & 8 \\
\hline APP & 10 & 7 & 7 & 6 & 6 & 5 \\
\hline B2A & 10 & 9 & 7 & 6 & 5 & 5 \\
\hline B3A & 10 & 9 & 6 & 5 & 4 & 4 \\
\hline DMP & 10 & 9 & 6 & 6 & 5 & 5 \\
\hline T2A & 10 & 9 & 6 & 5 & 4 & 4 \\
\hline T3A & 10 & 11 & 7 & 5 & 4 & 4 \\
\hline
\end{tabular}


Supplementary Figures

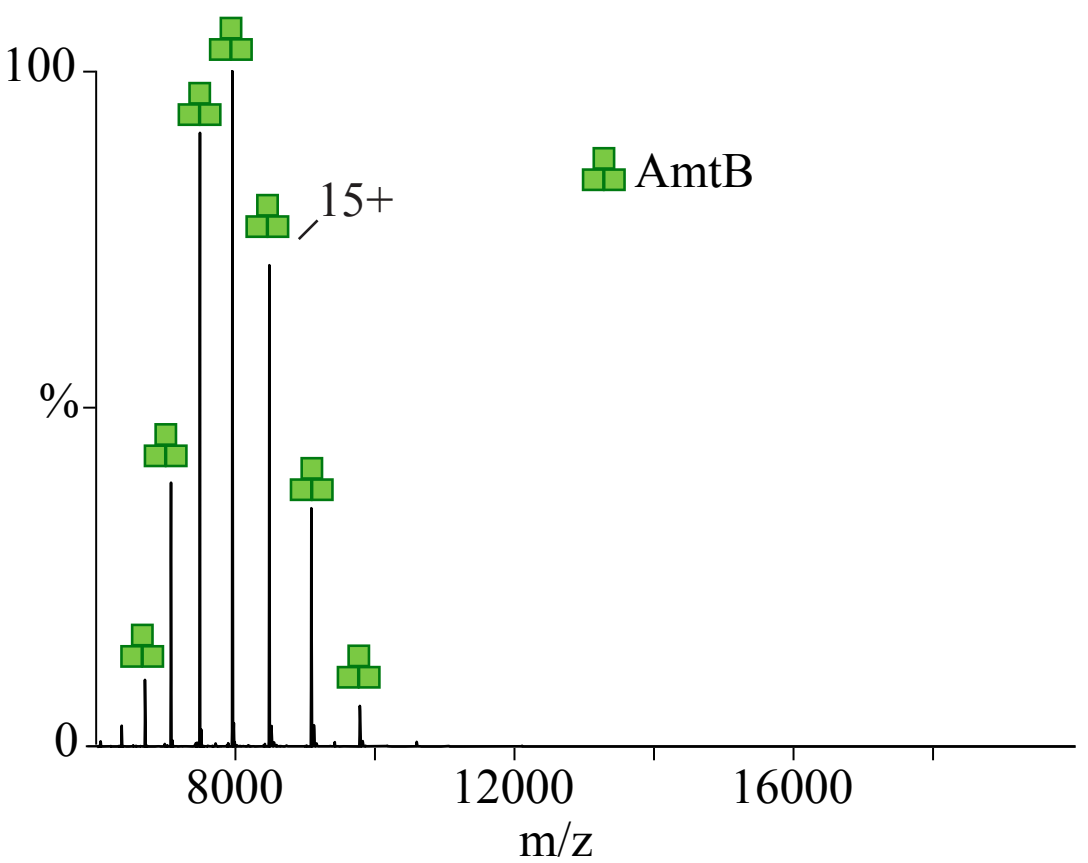

Figure S1. Native mass spectrum of AmtB in $200 \mathrm{mM} \mathrm{AA}$ with $0.5 \%(\mathrm{v} / \mathrm{v}) \mathrm{C}_{8} \mathrm{E}_{4}$ collected on the UHMR, no additional charge reducing reagent added. 

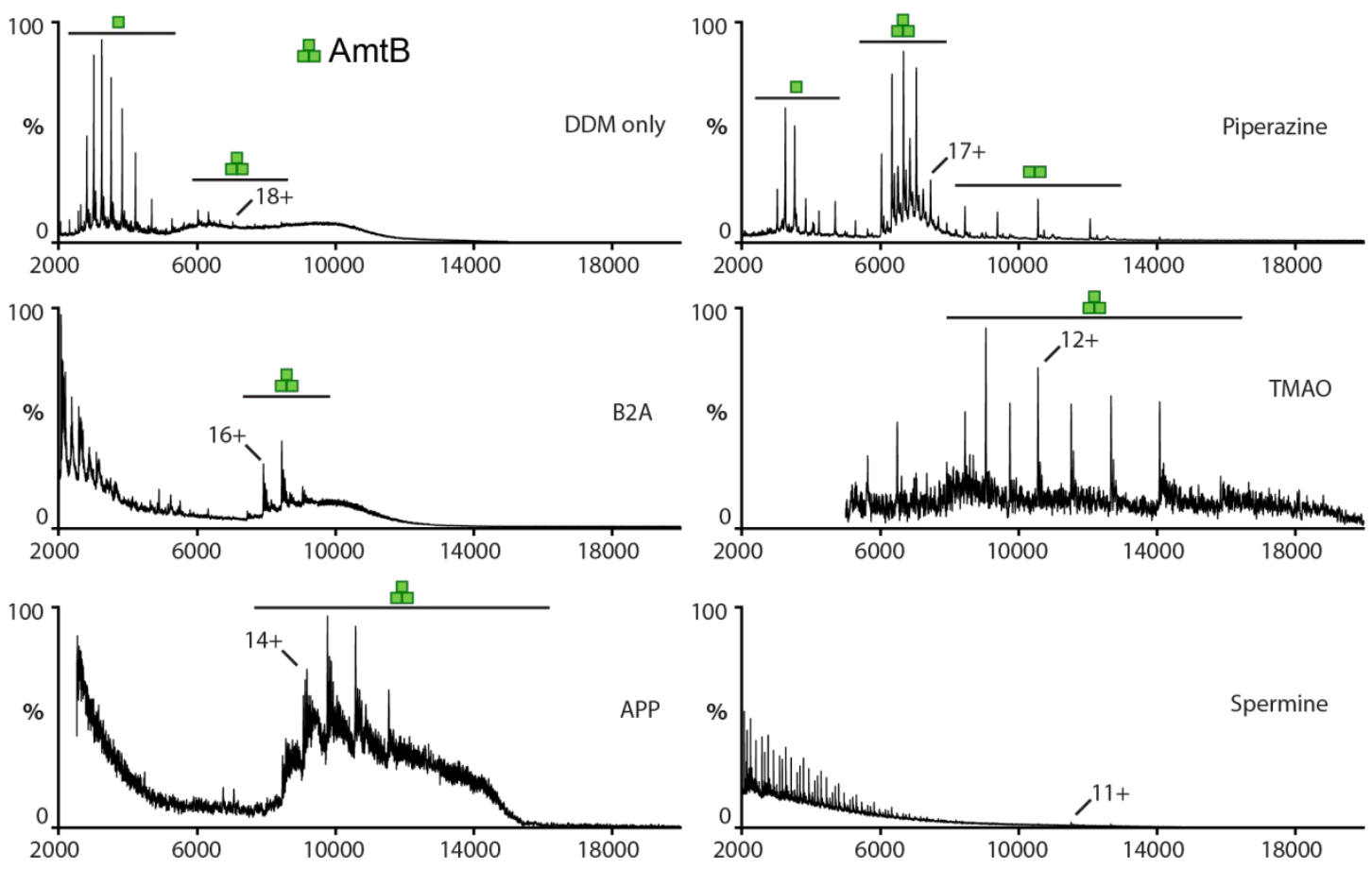

Figure S2. Native mass spectrum of AmtB solubilized by DDM with added charge reducing agents. Shown are the mixtures that generated interpretable mass spectra. Other agents used in this study causes poorly resolved peaks when mixed with AmtB in DDM and are not shown. Note that AmtB trimers readily falls apart with insufficient charge reduction. 

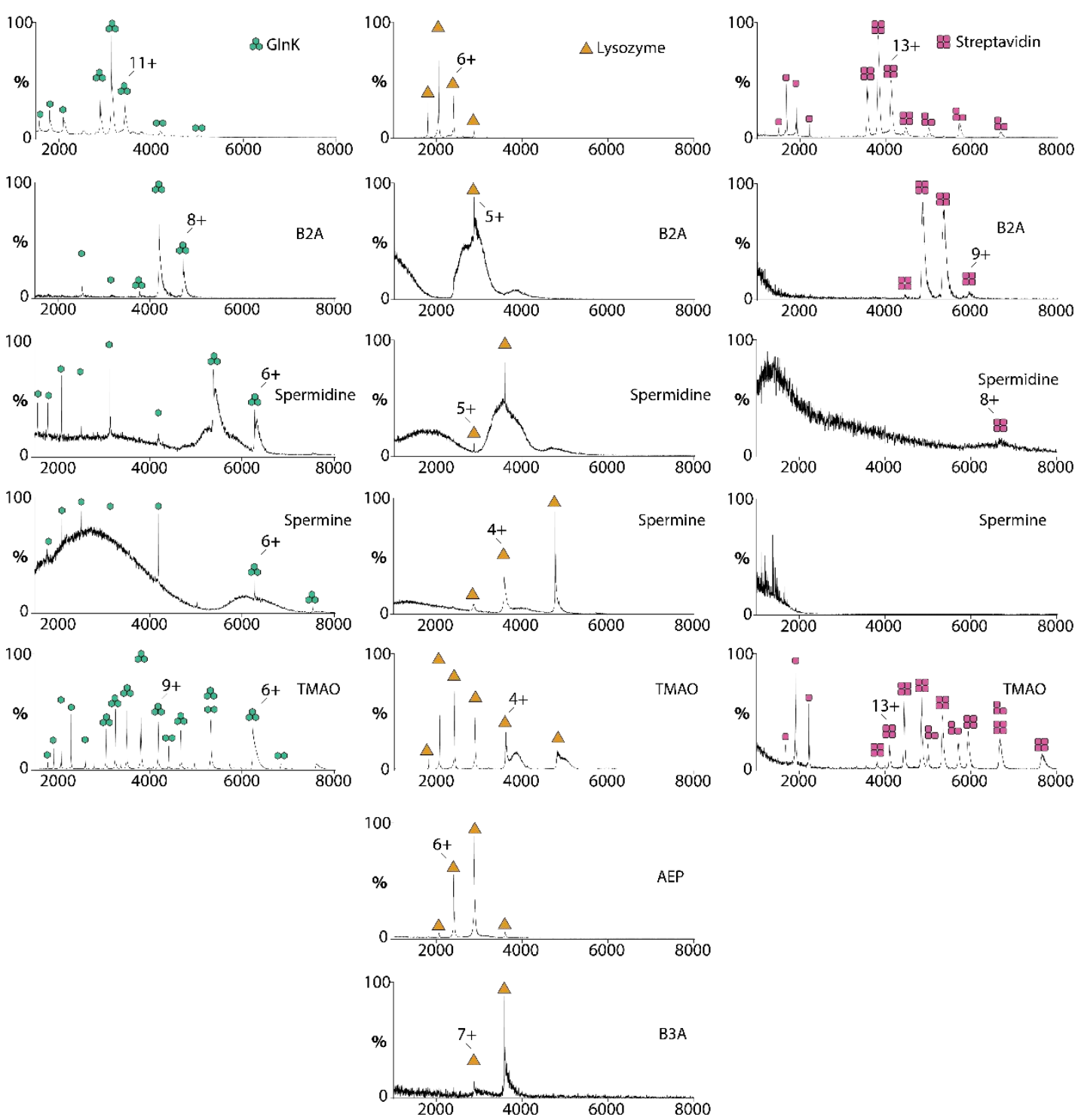

Figure S3. Native mass spectra showing variable charge reduction effects on soluble proteins. Glnk (Left column), Lysozyme (middle column) and Streptavidin (right column) show representative spectra with decipherable mass species. Top row show representative spectra for respective soluble proteins in $200 \mathrm{mM}$ ammonium acetate, $\mathrm{pH} 7.4$. 

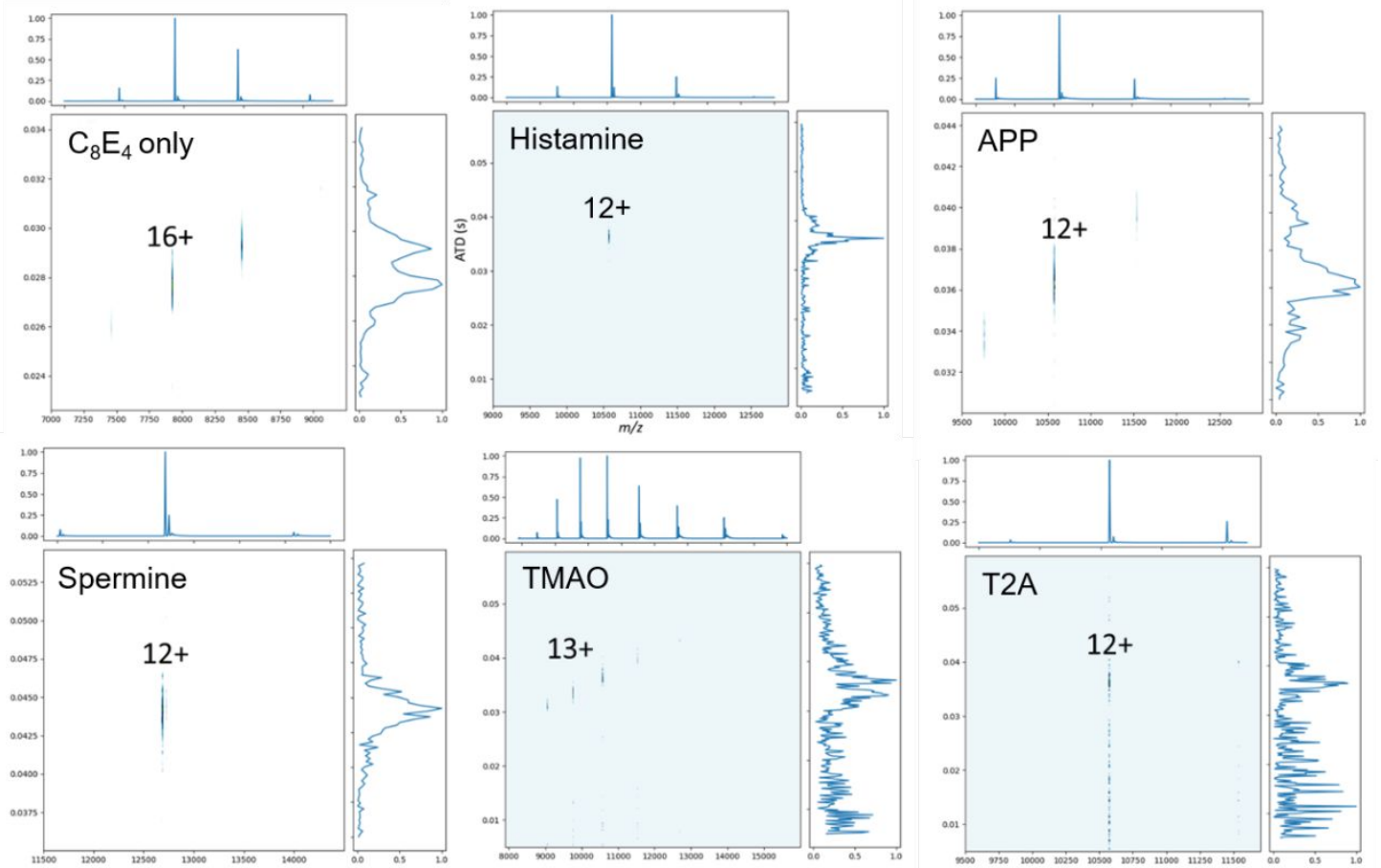

Figure S4: MS, 2D ATD(s) vs $\mathrm{m} / \mathrm{z}$, and ATD plots for all molecules analyzed via the FT-IM-PF-DT Orbitrap Platform. 


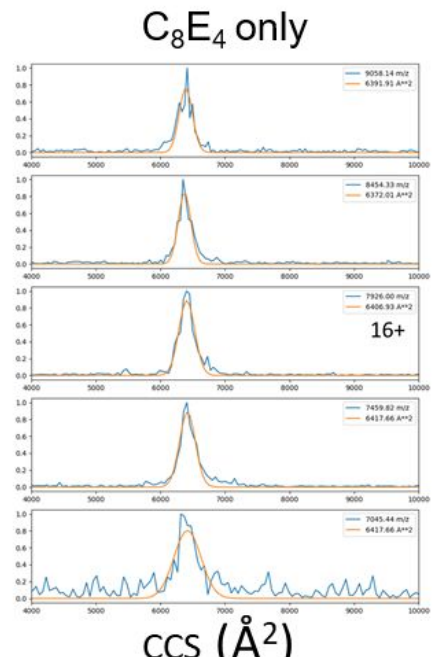

T2A
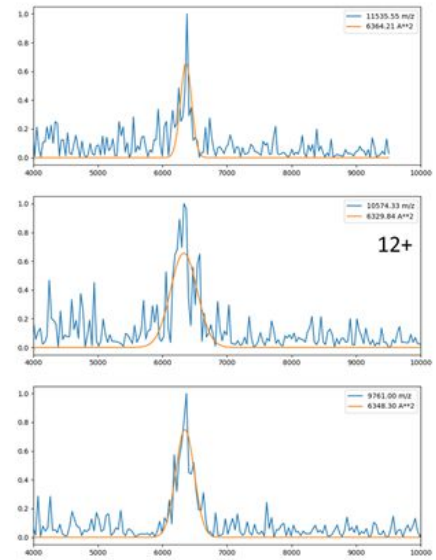

$\operatorname{ccs}\left(\AA^{2}\right)$

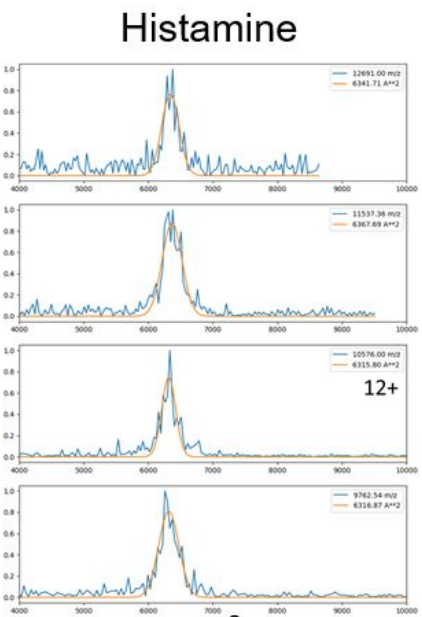

$\operatorname{ccs}\left(\AA^{2}\right)$

Spermine
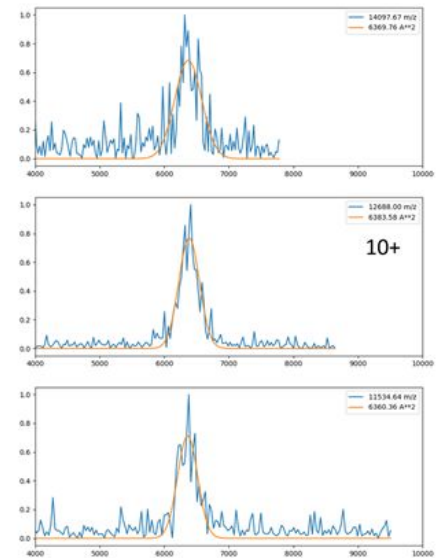

$\operatorname{ccs}\left(\AA^{2}\right)$
TMAO

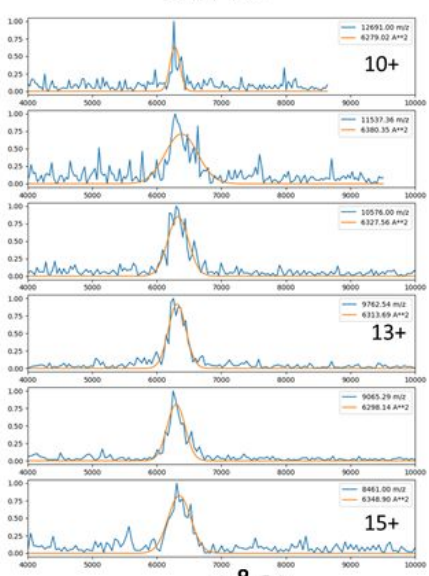

$\operatorname{ccs}\left(\AA^{2}\right)$

APP
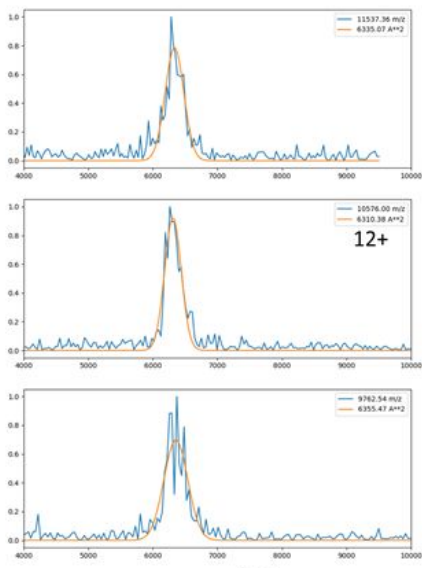

$\operatorname{ccs}\left(\AA^{2}\right)$

Figure S5: Raw data and Gaussian fitted CCS peaks for all molecules analyzed via the FT-IM-PF-DT Orbitrap Platform 


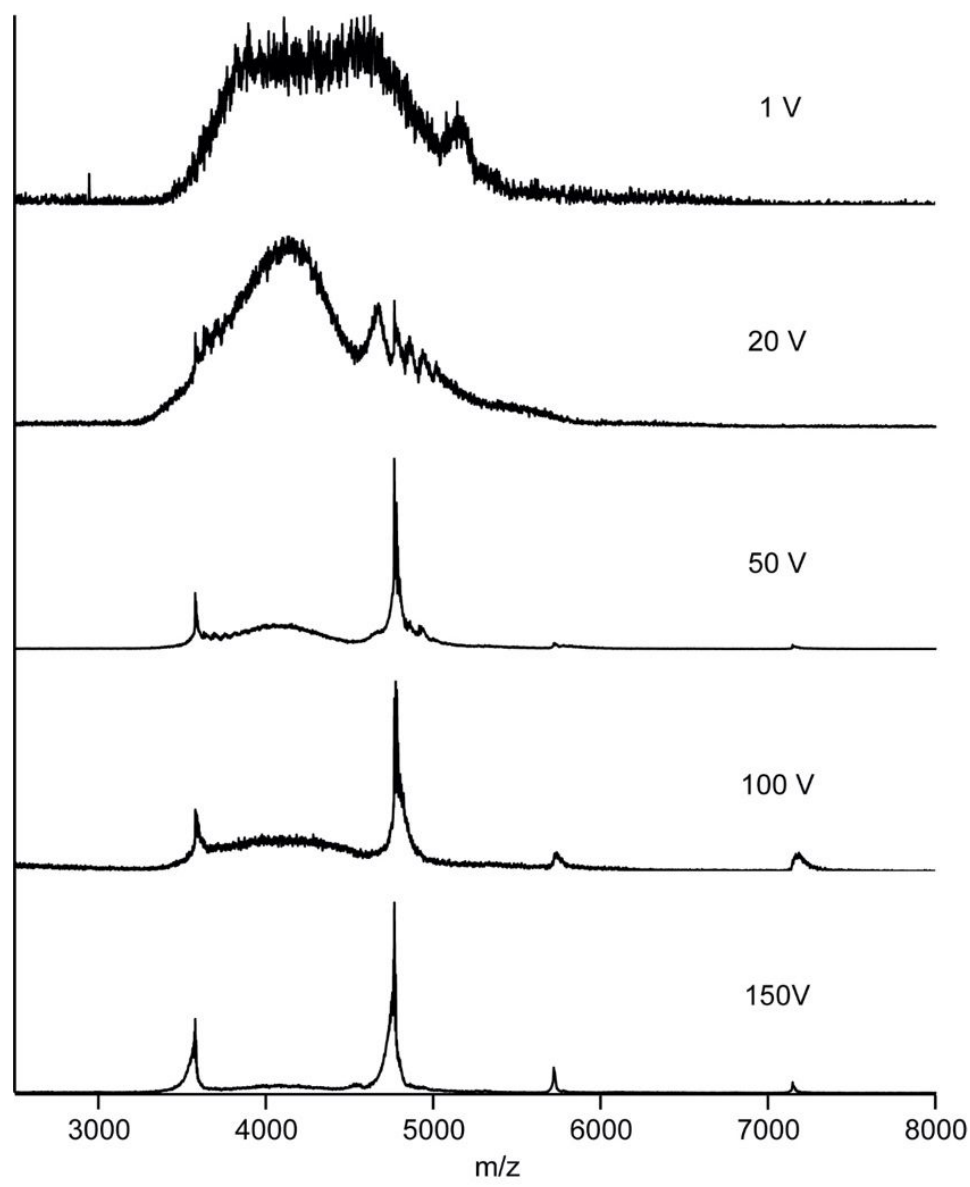

Figure S6: Mass spectra of charge-reduction of lysozyme by $50 \mathrm{mM}$ spermine with increasing collision energy. 


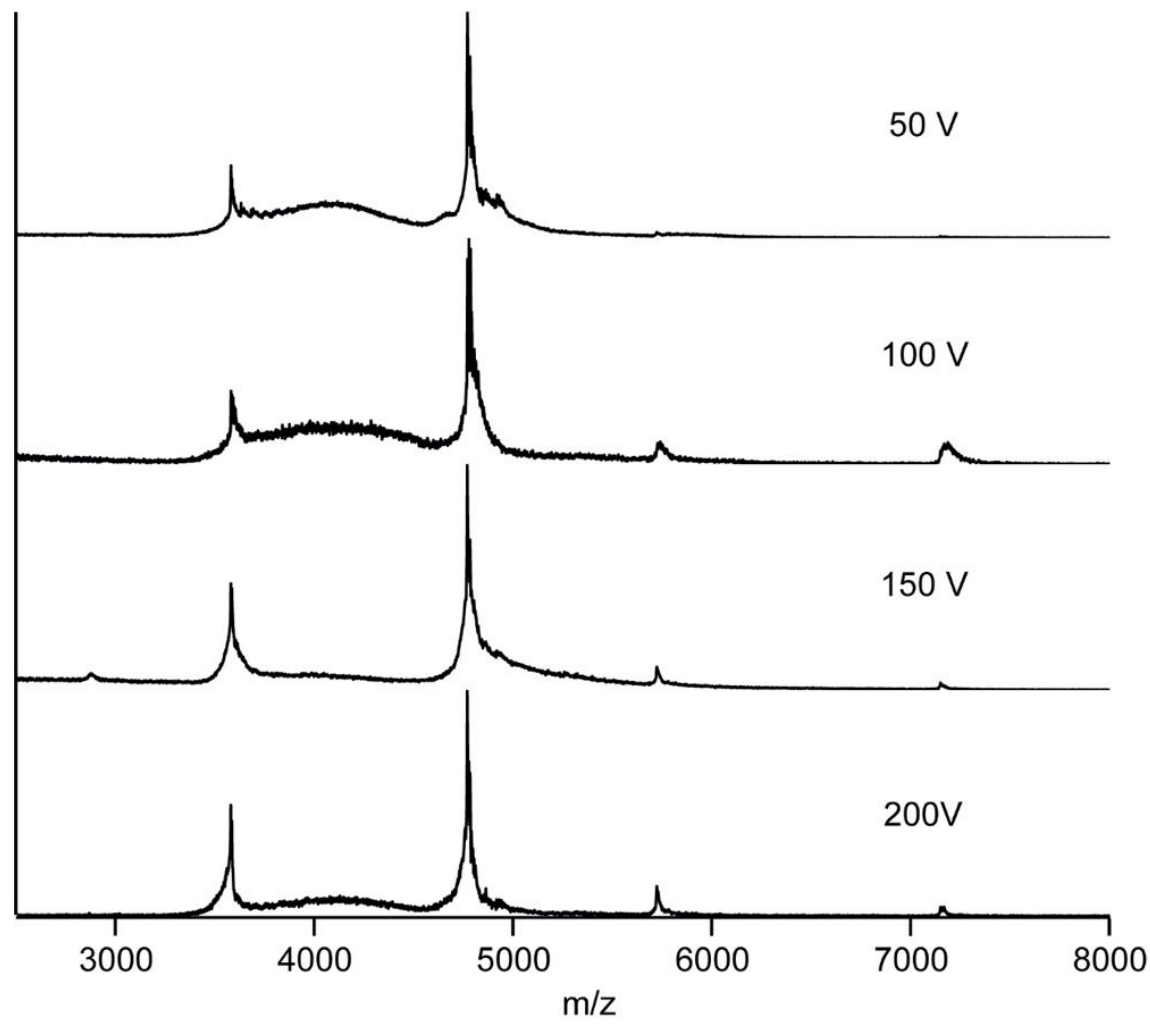

Figure S7: Mass spectra of charge-reduction of lysozyme by $50 \mathrm{mM}$ spermine with increasing cone voltage. 


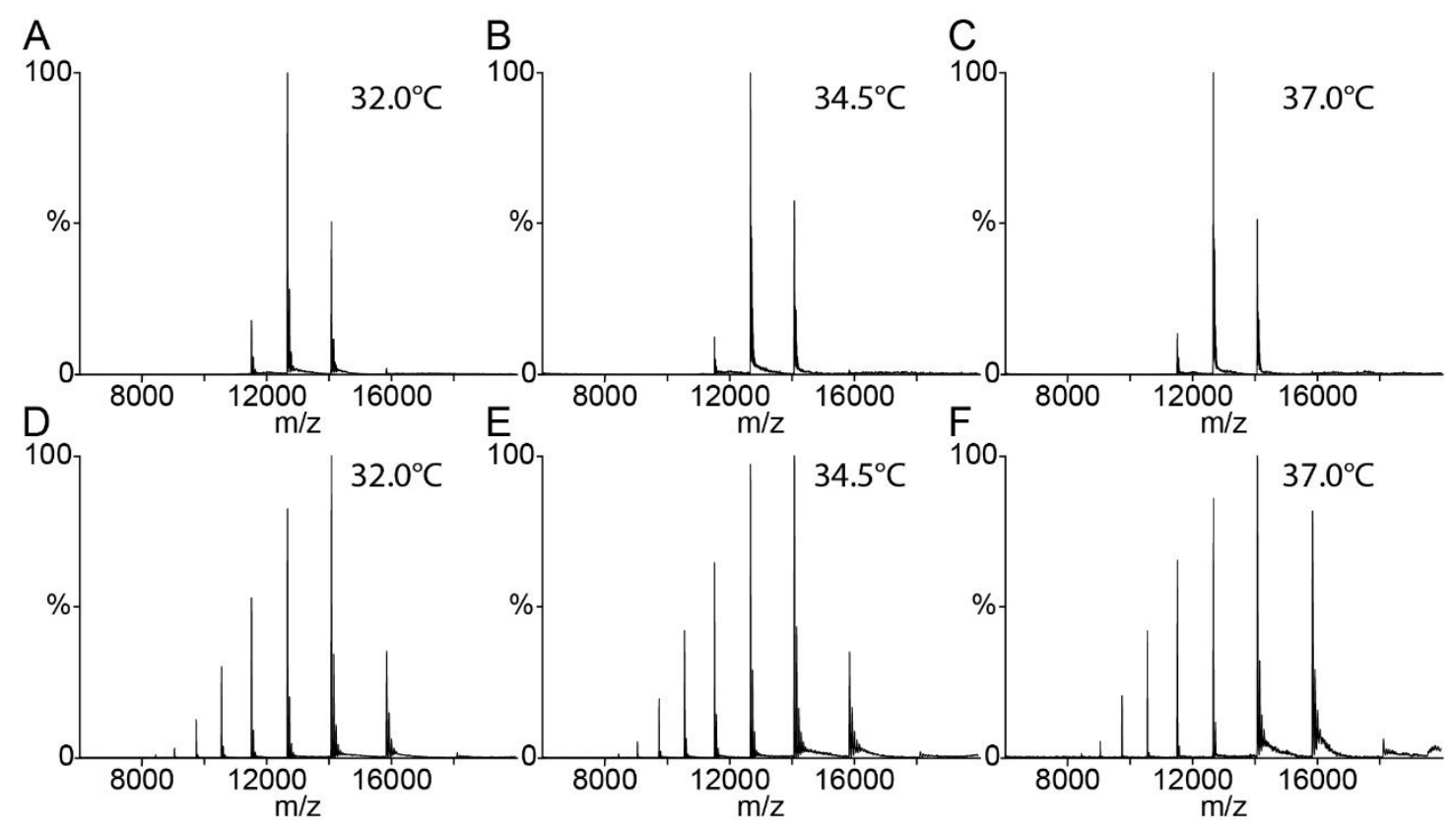

Figure S8: A-C) Mass spectra of $A m t B$ charge reduced by $100 \mathrm{mM}$ spermine with solution temperature at $(A) 32.0^{\circ} \mathrm{C}(B) 34.5^{\circ} \mathrm{C}(\mathrm{C}) 37.0^{\circ} \mathrm{C}$. D-F) Mass spectra of AmtB charge reduced by $100 \mathrm{mM}$ TMAO with solution temperature at (D) $32.0^{\circ} \mathrm{C}(\mathrm{E})$ $34.5^{\circ} \mathrm{C}(\mathrm{F}) 37.0^{\circ} \mathrm{C}$. 